\title{
Bidirectional Benign Regulation of Acupuncture in the Treatment of Stroke
}

\author{
Long He ${ }^{1,2}$, Honglong Wu1,2, Anlai Liu' ${ }^{1}$, Meikui Zhang1,2* \\ ${ }^{1}$ Shaanxi University of Chinese Medicine, Xianyang 712046, Shaanxi Province, China \\ ${ }^{2}$ Chinese PLA General Hospital Telemedicine Centre, Beijing 100853, China \\ *Corresponding author: Meikui Zhang, zmk301@126.com
}

\begin{abstract}
Acupuncture is a traditional Chinese medicine therapy originated in China. In recent years, acupuncture is widely used in the field of neurological diseases, especially in the acute stage of stroke. Its mechanism of action is becoming clearer; one of the possible mechanisms is its bidirectional benign regulation.
\end{abstract}

Keywords: Stroke; Acupuncture; Bidirectional benign regulation; Brain network; Clinical treatment

Publication date: November 2021; Online publication: November 30, 2021

\section{Introduction}

There is no such concept of "bidirectional benign regulation" in ancient Chinese medical literature. Huangdi Neijing - The Numinous Pivot recorded that the key to using needles is to know how to regulate Yin and Yang. This may be the earliest theoretical prototype of the bidirectional benign regulation in ancient Chinese literature. After a long history of exploration and practice, in 1977, Yaguang Liu from the Chinese Academy of Sciences took the lead in proposing the concept of "bidirectional benign regulation" in Discussion on the Theoretical Problems of New Medicine from the Perspective of Molecular Biology. In 1980, Xiaoping Zhang from Anhui University of Chinese Medicine put forward that acupuncture has a wide range of bidirectional regulatory effects on the nervous system, endocrine system, and immune system as well as defined this effect as the "bidirectional benign regulation"; Longxiang Huang from the China Academy of Chinese Medicine Sciences defined the bidirectional regulatory effect as the effect that acupuncture has in returning the body to equilibrium from a state deviated from a positive or negative direction. When it approaches or reaches the normal value, the effect will stop and there will be no such event of overcorrection. With the progress of acupuncture research, the theory, mechanism, and content of the bidirectional benign regulation are constantly enriched and improved, especially in the treatment of stroke.

\section{Bidirectional benign regulation of acupuncture on hemorrhagic stroke and ischemic stroke}

One of the connotations of the bidirectional benign regulation of acupuncture is that taking the same acupoints within a certain range can result in positive therapeutic effects on different diseases; Neiguan point and Shuigou point are often used in acupuncture for the treatment of stroke. Recent studies have shown that these two points have curative effects on acute hemorrhagic stroke and ischemic stroke. In the treatment of acute cerebral hemorrhage by acupuncturing the Shuigou and Neiguan points in an animal model, Jiawei Han and other researchers found that it reduces the expression of caspase-3 and caspase-9 protein, slows down and inhibits neuronal apoptosis mediated by the caspase family, reduces brain tissue 
damage after hemorrhage, as well as protects neuronal function for 6 hours ${ }^{[1]}$. Xiaozhuo Sun and other researchers used the "bird-pecking needling" method on the Shuigou point, while they used the twirling and draining method in needling the Neiguan point. It was found that after intracerebral hemorrhage, upon fast frequency ( 3 times per second) acupuncture for 60 seconds, once a day, continuously for 5 days, the expression of AQP-4 protein in brain tissue reduces, thus relieving intracranial edema; in the acupuncture group, the ultrastructure of the brain tissue under electron microscope showed that the survival rate of normal neurons was higher ${ }^{[2]}$. In terms of ischemic stroke, research has shown that acupuncturing on Neiguan, Shuigou, and other acupoints can upregulate $\beta$-catenin and downregulate Axin 2 and mRNA, thus activating Wnt/ $\beta$-catenin signaling pathway and promoting the expression of vascular endothelial growth factor (VEGF), so as to improve the stability of blood-brain barrier (BBB) ${ }^{[3]}$. In another study, Guangxia $\mathrm{Ni}$ and other researchers studied the treatment of acute ischemic stroke (AIS) by acupuncturing on Neiguan, Shuigou, and other acupoints in combination with rtPA thrombolysis; acupuncture was initiated 2 hours after cerebral ischemia, while rtPA thrombolysis was initiated 6 hours later. Compared with the thrombolysis group, the VEGF and basic fibroblast growth factor (bFGF) antibodies of the combined group were upregulated and the expression of endostatin (ES) to promote angiogenesis was downregulated, thus prolonging the thrombolysis time window up to 6 hours ${ }^{[4]}$.

\section{Bidirectional benign regulation of acupuncture on the brain network link after stroke}

The human cerebral cortex comprises of about $10^{11}$ neurons, which are connected by $10^{15}$ connection points, forming a very sparse but complex network that can integrate real-time performance. This kind of network is a "small world network" [5]; specifically, the brain area is used as a node, and the biological function cooperation between different brain areas is used as a connection, thus forming a dense density. The short connection and the relatively sparse long connection can effectively integrate information in real time and enable information to be transmitted between multiple systems as well as further complete the cooperative operation between the functions of different brain regions ${ }^{[6-8]}$. With the progress of functional magnetic resonance imaging (fMRI), magnetoencephalography (MEG), positron emission tomography (PET), electroencephalography (EEG), functional near-infrared spectroscopy (fNIRS), and other technologies, the physiological phenomenon of cerebral function lateralization is now well known. Many studies gradually found that there is an imbalance of cerebral function lateralization in many diseases. After stroke, the coexistence of complex internal excitation and inhibition in the brains of these patients indicates that it is possible for the bidirectional adjustment of cerebral function lateralization through acupuncture, and one of the possible mechanisms of bidirectional benign regulation is the regulation of the brain network. The main mechanisms involve in acupuncture are endocrine regulation, immune regulation, and neural regulation. The bidirectional regulation of acupuncture on the brain network includes neural regulation but it is not only restricted to neural regulation. Previous fMRI studies have shown that a large number of neurons in the human brain are active and linked in a certain functional network in the resting state, which is a manifestation of the basic neural function of the human brain. In 2001, Raichle confirmed that there is a similar basic neural network in the resting brain and proposed the concept of default mode network (DMN) in the human brain ${ }^{[9]}$. Drummond believes that this kind of brain network is recognized as the most basic but important ${ }^{[10]}$. The theory of brain network opens up a macro perspective for exploring the bidirectional benign regulation mechanism of acupuncture. Xiao Han and Caihong $\mathrm{Fu}$ found that acupuncturing the Yanglingquan point in stroke patients can bidirectionally configure the key nodes of the brain network, turn over the relationship between the brain on the diseased side and the brain network on the healthy side, as well as improve limb dysfunction in these patients ${ }^{[11,12]}$. Sizhu Jiang treated stroke patients with scalp acupuncture and observed the changes of their brain network with fMRI and DTI. The results showed that 
acupuncture can enhance the functional connectivity (FC) of the brain on the diseased side, weaken the abnormal brain FC of the cerebral hemisphere on the healthy side, and promote the brain FC of the patient, in order to gradually balance the healthy side and the diseased side, thus reducing the defect in the limb function of stroke patients ${ }^{[13]}$.

\section{Bidirectional benign regulation of acupuncture and cerebral function lateralization after stroke}

With the progress of medical techniques such as fMRI, MEG, PET, EEG, fNIRS, and so on, the physiological phenomenon of cerebral function lateralization is now well known. With the progress of research, it has been found that the lateralization of cerebral function occurs in many diseases, and in the brain of patients with stroke, there is a coexistence of complex internal excitation and inhibition. In a normal right-handed person (left dominant hemisphere), there is more activity in the left hemisphere compared to the right. In Figure 1, the cerebral cortex suppresses each other through the neural pathway at the corpus callosum, forming a physiological balance. Assuming that an individual has a focal stroke in the left hemisphere as shown in Figure 2, the function of the left hemisphere is "struck," and there is reduced activity in the left hemisphere. The inhibition of the right hemisphere weakens as the right hemisphere loses inhibition from the left hemisphere and becomes more active. As a result, the inhibitory effect on the left hemisphere is also enhanced. At present, studies have shown that acupuncture can bidirectionally adjust the cerebral lateralization function. The theory of "cerebral function lateralization" explains the bidirectional regulatory effect of acupuncture very well from the perspective of neural regulation. It is possible for cerebral function to lateralize physiologically through acupuncture. This may be due to one of the mechanisms of bidirectional regulation in acupuncture ${ }^{[14]}$. Jiang Sizhu and other researchers believe that the function of the injured side decreases, and the functional compensation on the healthy side results in increased excitability and forms an abnormal lateralization pattern; that is to say, cerebral function lateralization in the physiological state before stroke does not conflict with the cerebral function lateralization in the pathological state after stroke. Based on this, the research by Jiang Sizhu and other researchers showed that scalp acupuncture enhances the functional connection of the injured cerebral hemisphere and effectively weakens the abnormally enhanced functional connection in the contralateral cerebral hemisphere; additionally, it promotes the normalization of the movement pattern of stroke patients in the process of rehabilitation. It can be seen that the effect of scalp acupuncture is characterized by the bidirectional adjustment of the functional connection in the brain, tending to a state of physiological lateralization. The study showed that acupuncture has obvious bidirectional regulation under the premise of the same acupuncture point and the same amount of stimulation, which can increase or decrease the excitability of different regions of the brain at the same time. EEG, fNIRS, and fMRI also objectively showed that the bidirectional adjustment of cerebral function to physiological lateralization after acupuncture may be one of the mechanisms of "bidirectional regulation" in acupuncture ${ }^{[13]}$. Zheng Zhong and other researchers believe that acupuncture can exert a biological effect similar to bidirectional neural regulation ${ }^{[15]}$. The main mechanism of acupuncture is endocrine regulation, immune regulation, and neural regulation. The bidirectional regulation of acupuncture on the brain network includes neural regulation but is not only restricted to neural regulation. 


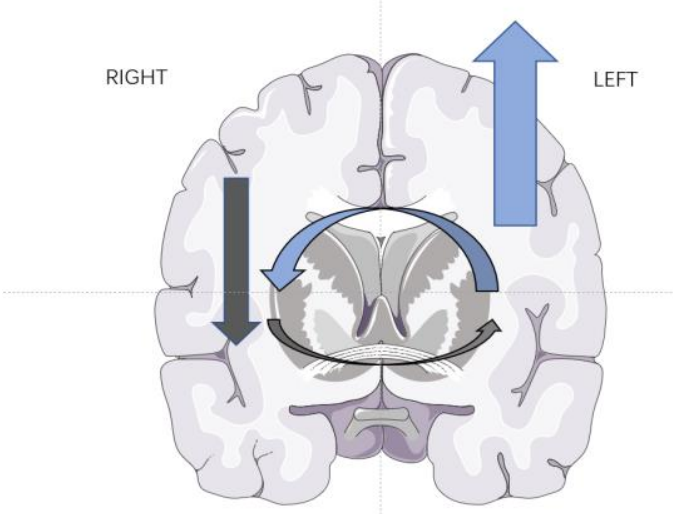

Figure 1. Brain of a normal person

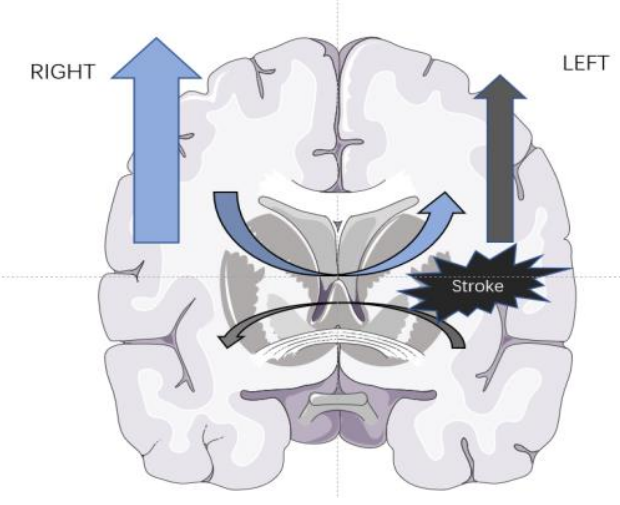

Figure 2. Brain of a stroke patient

\section{Discussion}

The complexity of brain function determines the complexity of the mechanism of acupuncture in the treatment of brain diseases. Bidirectional benign regulation is one of the characteristics of acupuncture, and it is also one of the diversified mechanisms of acupuncture in the treatment of stroke. Acupuncture and moxibustion have become an independent medical department and medical discipline in China, but in the medical circle of other countries, acupuncture is classified as complementary and alternative medicine (CAM). In consideration of the progress and development of acupuncture in the field of neuroscience in recent years, acupuncture should not only become the mainstream discipline and the main traditional Chinese medicine treatment in China, but also the main medical treatment in the field of neuroscience all over the world, so as to benefit more stroke patients.

\section{Disclosure statement}

The authors declare that there is no conflict of interest.

\section{References}

[1] Han J, Yang J, Chang L, et al., 2020, Effect of Acupuncture "Shuigou" "Neiguan" on Expression of Apoptosis Related Factors in Cerebral Tissue around Hematoma in Rats with Cerebral Hemorrhage. Acupuncture Research, 45(10): 812-817.

[2] Sun X, Yang Z, Meng Z, 2109, Protective Mechanism of Blood-brain Barrier in Rats with Cerebral Hemorrhage by Different Duration of Acupuncture. China Journal of Traditional Chinese Medicine and Pharmacy, 34(11): 5123-5126.

[3] Li G, Li X, Dong J, et al., 2021, Effects of Electroacupuncture on Neurovascular Units and Wnt/ $\beta$ catenin Signaling Pathway in Rats with Cerebral Ischemia. Acupuncture Research, 46(02): 87-94.

[4] Chang S, Zhang X, Zhang A, et al., 2021, Study on Acupuncture Promoting Angiogenesis and Prolonging Thrombolysis Time Window of Cerebral Infarction. Acupuncture Research, : 1-10.

[5] Sporns O, Zwi JD, 2004, The Small World of the Cerebral Cortex. Neuroinformatics (Neuroinformatics), 2(2): 145-162.

[6] Sporns O, Chialvo DR, Kaiser M, et al., 2004, Organization, Development and Function of Complex Brain Networks. Trends in Cognitive Sciences (Trends Cogn Sci), 8(9): 418-425.

[7] Achard S, Salvador R, Whitchere B, et al., 2006, A Resilient, Low-Frequency, Small-World Human Brain Functional Network with Highly Connected Association Cortical Hubs. J Neurosci, 26(1): 63- 
72.

[8] Sporns O, Honey CJ, 2006, Small Worlds Inside Big Brains. PNAS, 103(51): 19219-19220.

[9] Raichle ME, MacLeod AN, Snyder AZ, et al., 2001, A Default Mode of Brain Function. Proceedings of the National Academy of Sciences of the United States of America, 98(2): 676-682.

[10] Drummond SPA, Walker M, Almklov E, et al., 2013, Neural Correlates of Working Memory Performance in Primary Insomnia. Sleep, 36(9): 1307-1316.

[11] Han X, 2019, Study on Brain Effect Mechanism of Acupuncture Intervention in Stroke Based on Whole Brain Functional Network. Beijing University of Chinese Medicine, 119.

[12] Fu C, 2016, Multiple Granger Causality Analysis of Yanglingquan Acupuncture on Resting Brain Network Response Characteristics of Stroke Hemiplegia. Beijing University of Chinese Medicine, 141.

[13] Jiang S, 2017, Study on the Mechanism of Scalp Acupuncture in Stroke Hemiplegia Based on Resting State fMRI and DTI. Beijing University of Chinese Medicine, 122.

[14] Wang D, Zhang B, Deng R, et al., 2021, Effects Analysis on "Lateralization of Brain" Theory and the "Two-way Regulation" of Acupuncture and Moxibustion. Journal of Clinical Acupuncture and Moxibustion, 37(02): 1-5.

[15] Zheng Z, Zou K, Yang C, et al., 2015, On the Rise and Fall Theory of Qi from the Perspective of Brain Asymmetry Variation of Mental Diseases. Journal of Chengdu University of Traditional Chinese Medicine, 38(4): 6. 